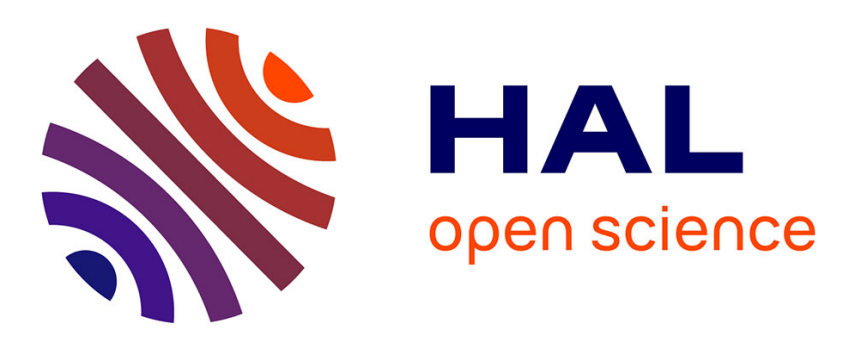

\title{
Air Temperature Fields inside Refrigeration Cabins: A Comparison of Results from CFD and ANN modelling
}

\author{
C. Conceição António, C.F. Afonso
}

\section{To cite this version:}

C. Conceição António, C.F. Afonso. Air Temperature Fields inside Refrigeration Cabins: A Comparison of Results from CFD and ANN modelling. Applied Thermal Engineering, 2011, 31 (6-7), pp.1244. 10.1016/j.applthermaleng.2010.12.027 . hal-00723973

\section{HAL Id: hal-00723973 \\ https://hal.science/hal-00723973}

Submitted on 16 Aug 2012

HAL is a multi-disciplinary open access archive for the deposit and dissemination of scientific research documents, whether they are published or not. The documents may come from teaching and research institutions in France or abroad, or from public or private research centers.
L'archive ouverte pluridisciplinaire HAL, est destinée au dépôt et à la diffusion de documents scientifiques de niveau recherche, publiés ou non, émanant des établissements d'enseignement et de recherche français ou étrangers, des laboratoires publics ou privés. 


\section{Accepted Manuscript}

Title: Air Temperature Fields inside Refrigeration Cabins: A Comparison of Results from CFD and ANN modelling

Authors: C. Conceição António, C.F. Afonso

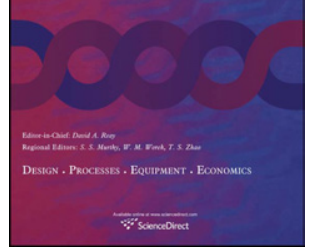

PII:

S1359-4311(10)00543-0

DOI:

10.1016/j.applthermaleng.2010.12.027

Reference: $\quad$ ATE 3351

To appear in: Applied Thermal Engineering

Received Date: 19 February 2010

Revised Date: 13 December 2010

Accepted Date: 16 December 2010

Please cite this article as: C. Conceição António, C.F. Afonso. Air Temperature Fields inside Refrigeration Cabins: A Comparison of Results from CFD and ANN modelling, Applied Thermal Engineering (2010), doi: 10.1016/j.applthermaleng.2010.12.027

This is a PDF file of an unedited manuscript that has been accepted for publication. As a service to our customers we are providing this early version of the manuscript. The manuscript will undergo copyediting, typesetting, and review of the resulting proof before it is published in its final form. Please note that during the production process errors may be discovered which could affect the content, and all legal disclaimers that apply to the journal pertain. 


\title{
Air Temperature Fields inside Refrigeration Cabins: A Comparison of Results from CFD and ANN modelling
}

\author{
Conceição António, C. and Afonso, C.F. \\ Faculdade de Engenharia, University of Porto \\ R. Dr. Robero Frias, 4200-465 Porto \\ Portugal \\ Email: cantonio@fe.up.pt
}

\begin{abstract}
In refrigerated spaces, the inside air is cooled by a heat sink operating either by forced or natural convection. The last situation is more frequently used in small apparatus, such as domestic household refrigerators. The inside air temperature is not usually monitored in these refrigerated spaces. Therefore, knowledge of the air temperature field inside of these units is limited and large air temperature gradients often exist that can put the stored products at risk. This work studies temperatures in a commercial household refrigerator that were monitored with thermocouples located at several points. The measured temperatures were then compared with those obtained from two different simulation tools: the Fluent code and another method based on an Artificial Neural Network with supervised learning performed using a Genetic Algorithm. Results lead to the conclusion that, at least in this case, the second tool produced a lower absolute error $(0.8 \mathrm{~K})$ when compared with the first $(1 \mathrm{~K})$ and yielded modelled inside air temperature fields that are more consistent with reality.
\end{abstract}

Key Words: Simulation, temperatures fields, refrigerator stores, Fluent, Artificial Neural Network 


\section{INTRODUCTION}

In refrigerated spaces, various perishable products are placed inside in an almost arbitrary order, based on the false principle that the inside air temperature is constant through the space. This is an erroneous premise because there are significant inside air temperature differences, either due to air circulation from the evaporator (natural or forced convection) or due to different thermal properties and/or respiration activity of the refrigerated products that interact with the neighbouring air. Because each perishable product has a typical conservation temperature for optimal longevity in good condition, it is necessary to understand the air temperature fields inside of the refrigeration stores.

Generally, the refrigeration spaces contain one thermostat with a bulb that senses the temperature inside, with the assumption that it is constant throughout the space, and this value then controls the compressor run time. Nothing is known about the air temperature in different locations within the space. Therefore, to obtain this knowledge, it is necessary to measure the inside air temperature in many places. This practice, however, is an expensive one and is not feasible in all stores.

To assist in solving this problem, the purpose of this work was to measure the inside air temperatures at selected discrete points in a commercial household refrigerator. The measured temperatures were then compared with values obtained from two different simulation tools, the Fluent code and another method based on an Artificial Neural Network (ANN) with supervised learning performed using a Genetic Algorithm (GA) supported by an elitist strategy. The aim was to obtain knowledge of the air temperature fields inside the refrigerated unit (in addition to the value measured by the thermostat).

The ANN method has been around since the 1940s [1], but its use has been recognised only recently (within the last twenty years), with applications in a large range of engineering fields. 
Several studies have been conducted in the field of thermal sciences, and they have mainly been concerned with optimisation of energy consumption in residential buildings due to solar heat gains, hot water heating and space cooling $[2,3,4,5]$. In terms of refrigeration systems, the ANN has also been used by several investigators for evaluation of the performance of vapour compression heat pumps [6,7], for automotive air conditioning [8], for modelling ejector-absorption refrigeration systems [9] and cascade refrigeration systems [10]. In the same domain, the commercial Fluent program has also been used for simulation and visualisation of temperature and velocity fields.

The results in this study showed that the ANN method is more precise, with a small absolute error between the measured and simulated values.

\section{TEST FACILITIES}

A commercially available household refrigerator was used for the tests [11], as seen in Figure 1. Previously calibrated copper-constantan thermocouples were located inside the cabin, three at three different levels in height, two on each vertical surface, one on each horizontal surface and also one at the outside laboratory. A total of twenty thermocouples were connected to a data acquisition system that read the temperatures at intervals of twenty seconds [12]. The refrigerator was empty during this time, and was disconnected from the grid at the beginning of the tests with the door open so that the inside and outside air temperatures were equal at the start. The door's shelves were open during the test in order to avoid close spaces inside the refrigerator. The tests began when the door was closed and the compressor was connected to the grid, starting its operation. Two distinct parts of the tests could be detected. The first one was a transient phase, where the compressor ran constantly until the thermostat detected a minimum temperature inside and the compressor was shut off. The second phase consisted of a steady state, where the compressor was switched on and off to maintain an average inside air temperature between 
a maximum and minimum air temperature established by the thermostat. The energy consumption of the compressor was also measured simultaneously.

\section{SIMULATION TOOLS}

As mentioned previously, two different simulation tools were used to simulate the inside air temperature fields in a commercial domestic refrigerator to gain as much knowledge as possible, and to compare the simulation tools in regards to the precision of the output. The following subsections describe the simulation tools used in this investigation.

\subsection{Fluent}

This commercially available simulation tool [13] uses the classic turbulent model $k-\varepsilon$ for the 3D simulations. It is based on the finite volumes method in which the equations of mass conservation and momentum are solved simultaneously, respectively:

$$
\begin{gathered}
\frac{\partial \rho}{\partial \tau}+\nabla(\rho \vec{v})=\int m \\
\frac{\partial}{\partial \tau}(\rho \vec{v})+\nabla(\rho \vec{v} \vec{v})=\nabla p+\nabla \stackrel{\bar{\Gamma}}{\Gamma}+\rho \vec{g}+\vec{F}
\end{gathered}
$$

These equations were integrated over each control volume centred on discrete points placed in the flow domain. Inside the refrigerator, a net of 432,000 control volumes were established with constant volume, except for those located close to the internal surfaces of the refrigerator where they were more refined. Therefore, the minimum and maximum values of the control volumes were $1.101 \times 10^{-8}$ and $7.155 \times 10^{-6} \mathrm{~m}^{3}$, respectively. The established convergence criteria were $10^{-2}$ for the mass conservation and $10^{-5}$ for the remaining equations.

For the simulation, the refrigerator was considered as a rectangular prism with the following internal dimensions: 1050x480x450 mm. The working fluid was air. 
The measured internal surface temperatures were used as boundary conditions input to the commercial Fluent code. A literature review [14] revealed procedures much like these, but instead of the measured boundary conditions used in this study, these values were considered as constant. From the results obtained, we concluded that the average error between the measured inside air temperatures and the simulated values was slightly larger than $1 \mathrm{~K}$ for all of the transient and steady phases of the test.

Figures 2 and 3 show the results obtained for the air temperature fields inside the refrigerator at the end of the transient phase and in a steady phase, respectively. The compressor was stopped in both situations. In the first case, the compressor had completely stopped and in the second case, it was about to start up.

As can be seen in Figure 2, the air temperatures decrease from the top to the bottom of the refrigerator, as expected. A higher temperature is noticeable close to the top right wall, but this is due to some inefficiency of the thermal insulation, as confirmed by the refrigerator producer. However, this inefficiency affects the air temperatures inside the unit. As can also be seen in the figure, there is a large stratification of the air temperatures and a noodle of lower temperature at the bottom location that cannot be explained by the measurements that were carried out. The temperature difference between the top and the bottom locations is approximately $5 \mathrm{~K}$.

In the steady phase of Figure 3, the compressor was about to start up. Therefore, the inside air temperatures are higher when compared with those of the previous situation, and the air temperatures are also observed to decrease from the top to the bottom locations. In this case, two spots can be seen at about midlevel heights that show higher temperatures in the surrounding air, without any apparent justification when compared with the measured temperatures. Because these spots are located at different positions as compared with Figure 1, they cannot be input to the thermocouple readings.

On the top of the plane there is a layer of lower temperatures caused by the heat transfer from the refrigerator to the freezer (at lower temperatures). 


\subsection{Artificial Neural Network (ANN)}

The Artificial Neural Network (ANN) method is based on a computational structure inspired by the biology of the human neural system, including attributes such as learning, thinking, memorising, remembering, rationalising and problem solving. The ANN is made up of simple and highly interconnected nodes called neurons. One artificial neuron can modify its behaviour in response to the environment where it is located.

ANNs are widely accepted as a technology that offers an alternative way to treat complex and ill-defined problems. ANNs can learn from experiments, are tolerant of noisy and incomplete data, and are able to deal with non-linear problems. Once trained, they can perform prediction and generalisation at high speed. ANN applications have been reported in energy fields, including heating, ventilating and air-conditioning, solar radiation, modelling and control of power generation systems, load forecasting and prediction, and refrigeration [14-17]. However, the study of temperature fields inside refrigerated spaces is a new focus.

An ANN was specially developed for the case under study. The input data to the ANN are the Cartesian coordinates in the refrigerator, one time instant for the transient phase and one time instant for the stationary phase of the refrigeration process. The output data are the simulated air temperature fields corresponding to the transient and steady phases of the process for the instants considered for the input data. The ANN model is based on a topology with three layers, one of which is hidden.

When a set of data (local coordinates and time instants) is introduced into the network, it adapts itself to obtain consistent output data (temperatures) through a process called learning. The model developed here is based on supervised learning. In this case, for a set of input data, there is a set of previously defined output temperatures. Therefore, the input data generates a set of output data that is compared with the measured values. The learning phase occurs if there are differences between the simulated output and the desired ones, as obtained experimentally and known a priori. In this case, the weights of the synapses and 
the biases of the ANN are adjusted to decrease that difference. For that, the input data must be linearly independent, which results in a system that can be solved if hidden nodes are introduced on the topology of the network. However, the hidden nodes also raise problems, such as an inability to recognise which of the weighted connections is responsible for the difference (error). To solve this problem, a methodology was developed that iteratively considers the output signal as an input signal and then fits the weights along all of the process. This process is known as back propagation. The input signals are modified through weights that represent the existing connections between nodes.

In our approach, the training of the learning process of the ANN is realised through a designed elitist Genetic Algorithm. Using a binary code format for the weights of the synapses and the biases of the ANN, it is possible to evaluate the fitness of each input/output pair based on the absolute error. Therefore, the main objective is to minimise this error. With this optimisation process, the ANN obtains a correct simulation of the temperatures using the experimental measurements.

\subsubsection{ANN Topology Definition}

The aim of this simulation tool is to investigate the performance of the Artificial Neural Network (ANN) in regards to the precision of the evolution of the inside air temperatures in a refrigerator. Each set of measured data is associated to an input vector and an output vector. The weighted links between the input nodes and the intermediate nodes, and between these and the output nodes, are designed by synapses. These connections establish the relationship between input data $O_{i}^{\text {inp }}$ and output data $O_{j}^{\text {out }}$.

In the developed ANN, the input data, $O_{i}^{i n p}$, are the Cartesian coordinates in the refrigerator, one time instant for the transient phase and one time instant for the stationary phase of the refrigeration process. The output data, $O_{j}^{\text {out }}$, are the simulated air temperatures fields corresponding to the transient and steady phases of the process for the instants considered for the input data. The ANN 
model is based on a topology with three layers, one of which is hidden. Figure 4 shows the topology of the ANN together with the input and output parameters.

As put forth, the set of data is converted in an input vector and on an output vector. These must be normalised to avoid numerical perturbations in the information process. Each pattern consisting of an input vector and an output vector needs to be normalised with the aim of avoiding error propagation during the ANN learning process. This is achieved using the following data normalisation:

$$
\bar{O}_{k}=\left(O_{k}-O_{\min }\right) \frac{O_{N \max }-O_{N \min }}{O_{\max }-O_{\min }}+O_{N \min }
$$

where:

$O_{k}$ is the real value of the variable before normalisation.

$O_{\min }$ and $O_{\max }$ are the minimum and the maximum values of $O_{k}$ in the data set to be normalised.

The input and output data are then normalised to values $O_{N \min }$ and $O_{N \max }$ such that $O_{N \text { min }} \leq \bar{O}_{k} \leq O_{N \text { max }}$. Depending on the input or the output data, different predefined values of $O_{N \min }$ and $O_{N \max }$ can be used.

Figure 5 represents the calculation process for a single $k$-th node (neuron), designed as a Process Element (PE). The sum of the modified signals (total activation) is modified through a function, designed as an Activation Function $F(x)$. The activation of node $k$ of the intermediate layer $(m=1)$ and output layer $(m=2)$ is obtained through sigmoidal functions of the following kind:

$$
F_{k}^{(m)}=\frac{1}{1+\mathrm{e}^{-\alpha^{(m)}} I_{k}^{(m)}}
$$

In this equation, $\mathrm{m}$ represents the activation zone (either hidden or output layers) and $I_{k}^{(m)}$ are the components of the vector $\mathbf{I}^{(m)}$ given by: 


$$
\mathbf{I}^{(m)}=\mathbf{W}^{(m)} \mathbf{O}^{(m)}+\mathbf{b}^{(m)}
$$

where:

$\mathbf{W}^{(m)}$ is the matrix of the weights of synapses associated with the linkage between the input and hidden layers $(m=1)$ or between the hidden and output layers $(\mathrm{m}=2)$.

$\mathbf{b}^{(m)}$ is the bias vector on hidden $(m=1)$ or output $(m=2)$ layers.

$\mathbf{O}^{(m)}$ is the input data vector for the hidden layer $(m=1)$ or the output layer $(m=2)$.

The weights of the synapses $w_{i j}^{(m)}$ and the biases in the neurons in the hidden and output layers $b_{k}^{(m)}$ are controlled during the learning process. The absolute error between experimental and numerical results is used to monitor the learning process with the aim of obtaining completeness of the model of the refrigeration process.

When a set of input data is introduced into the network, the network adapts to produce consistent results through a process designed by learning. Therefore, for a set of input data, there exist a predefined set of output data. Then, using the input data in the network, the model generates numerical output results that can be compared with the measured values and as such, the mean absolute error can be evaluated through the following equation:

$$
\operatorname{Error}(\mathbf{W}, \mathbf{b})=\frac{1}{2 N^{\exp }} \sum_{n=1}^{N^{\exp }}\left(\sum_{i=1}^{2}\left|\bar{O}_{i}^{\exp }-\bar{O}_{i}^{\text {out }}\right|\right)_{n}
$$

where:

$N^{\exp }$ is the available number of experimental measured values of the inside air temperatures 
$\bar{O}_{i}^{\text {out }}$ and $\bar{O}_{i}^{\exp }$ are the simulated and measured air temperatures, respectively.

The error obtained from equation (6) is back propagated into the artificial network. This means that, from output to input, the weights of the synapses and the biases can be modified until the error falls within a prescribed value.

The learning process will proceed if there is a difference between the simulated results and the desired values (known a priori through the measured experimental data). Then, the weight of the synapses $\mathbf{W}$ and the biases of the neurons of the hidden layer and of the output $\mathbf{b}$ are adjusted to reduce the differences (supervised learning). This adjustment happens in an optimisation process.

\subsubsection{ANN Learning Process based on Genetic Algorithm}

Genetic Algorithms (GAs) have been used with increasing frequency in a large variety of applications. These methods apply biological principles of survival-ofthe-fittest in computational algorithms. The GA uses a structured exchange of data to explore all regions of the domain and leads some operators to exploit potential areas. These methods are also able to deal with discrete optimum design problems and do not require derivatives of functions.

The use of GAs in ANN learning is a methodology that has been applied by some authors in industrial processes. In our approach, the objective is to explore some of the advantages of GAs in handling discrete input parameters associated in the refrigeration process as Cartesian coordinates, as well as the time instants in transient and steady phases. It is understood that the learning process is an optimisation procedure.

The adopted supervised learning process of the ANN based on a GA uses the weights of synapses $\mathbf{W}^{(m)}$ and the biases of neural nodes at the hidden and output layers $\mathbf{b}^{(m)}$ as design variables. A binary code format is used for these variables and the number of digits of each variable can be different. The bounds 
of the interval domain of the learning variables and the scaling parameter $\alpha^{(\mathrm{m})}$ influence the sensitivity of sigmoidal activation functions and must be controlled.

The optimisation problem formulation associated with the ANN learning process is based on the minimisation of the absolute error defined in equation (6). A regularisation term associated to biases in the hidden and output neurons are included in the fitness function for learning based on GA, with the aim of accelerating the convergence of the ANN learning process.

Since the evolutionary search is intended to maximise a global fitness function FIT that measures the ANN performance, the optimisation problem is defined as follows

$$
\text { Maximise } F I T=K-\left[\operatorname{Error}\left(\mathbf{w}^{(1)}, \mathbf{w}^{(2)}, \mathbf{b}^{(1)}, \mathbf{b}^{(2)}\right)+B\left(\mathbf{b}^{(1)}, \mathbf{b}^{(2)}\right)\right]
$$

where:

$K$ is an arbitrary constant

$B\left(\mathbf{b}^{(1)}, \mathbf{b}^{(2)}\right)$ is a regularisation function associated to the mean quadratic biases of the hidden and output layers and defined as

$$
B\left(\mathbf{b}^{(1)}, \mathbf{b}^{(2)}\right)=\left(B^{(1)}+B(2)\right)^{1 / 2}
$$

with

$$
\begin{aligned}
& B^{(1)}=\sum_{m=1}^{N^{\exp }}\left[\frac{1}{N^{\text {hid }}} \sum_{k=1}^{N^{\text {hid }}}\left(b_{k}^{(1)}\right)^{2}\right]_{m} \\
& B^{(2)}=\sum_{m=1}^{N^{\exp }}\left[\frac{1}{N^{\text {out }}} \sum_{k=1}^{N^{\text {out }}}\left(b_{k}^{(2)}\right)^{2}\right]_{m}
\end{aligned}
$$

and: 
$b_{k}^{(1)}$ and $b_{k}^{(2)}$, the associated biases in the nodes of the hidden and output layers respectively.

\subsubsection{Genetic Algorithm}

The Genetic Algorithm performs the learning process based on four operators (selection, crossover, replacement and elimination) as well as implicit mutation of similar individuals. The GA is the same method that has been used in previous industrial applications [8,9]. The algorithm is applied as follows:

Step1: Initialisation. The initial population $\mathrm{Pop}(0)$ is randomly generated.

Step 2: Selection. This operator selects the parents: one from the best-fitted group (elite) and another from the least-fitted one. This selection is done randomly with an equal probability distribution for each individual. The Crossover operator determines transfer of the entire population to an intermediate step where it is joined with the offspring.

Step 3: Crossover. The offspring genetic material is obtained using a modification of the "Parameterised Uniform Crossover" technique proposed by Spears and De Jong [18]. This is a multi-point combination technique applied to the binary string of the selected chromosomes. The offspring gene is selected in a biased way, given a defined probability for choosing the gene from the elite chromosome. The new offspring group is then joined to the original population.

Step 4: Replacement by similarity. A new ranking of the enlarged population is implemented according to individual fitness. This is followed by Elimination of solutions with similar genetic properties and subsequent Substitution by new randomly generated individuals.

Step 5: Elimination. The population is ranked again, followed by Elimination corresponding to the deletion of the worst solutions. The exclusion of individuals with low fitness and the natural death of old individuals are simulated by this operator. This elitist strategy enables the survival of parents and offspring together in an evolutionary process. At this point, the dimension of the 
population is smaller than that of the original one. The original-sized population will be recovered after inclusion of a group of new solutions obtained from the Mutation operator.

Step 6: Implicit Mutation. To avoid the appearance of local minima, a chromosome set group in which genes are generated in a random way is introduced into the population. This operation is called implicit mutation. The mutation operator guarantees the diversity of the population in each generation.

Step 7: Stop criterion. The stop criterion used in the evolutionary process is based on the relative variation of the mean fitness of a reference group during a fixed number of generations and the feasibility of the corresponding solutions. The size for the reference group is predefined [19].

\subsubsection{Simulation using ANN}

One important aspect of the ANN is the normalisation of the input and output data of the net. For the model built for the refrigerator, the data was normalised for the following domain: $[0.9,0.1]$. The learning process considered 17 pairs of input/outputs and measured temperatures associated with the numbers of the thermocouples distributed at several points inside the refrigerator $(x, y, z)$. An ANN with six nodes at the hidden layer was used. A population of 24 individuals is used in ANN learning based on GA with a mutation group of eight individuals. A binary code format with five digits is used for the weights of synapses and bias design variables of the ANN learning process. Figure 7 shows the evolution of mean absolute error along the ANN learning process based on GA. At the end of the learning process, one optimal ANN was obtained with an absolute error of $0.8 \mathrm{~K}$ between the simulated and measured temperatures for all of the experiment.

After the learning process, a simulation procedure was implemented based on the optimised ANN and designed to obtain the inside temperature field in a reference plane. The frontal plane located at the middle of the refrigeration cabin was selected. 
The results of the temperature fields as simulated with the ANN for the same plane and time instants considered for the Fluent code are shown in Figures 8 and 9, respectively. In both figures the results are obtained using ANN after its learning procedure (optimal topology) and introducing the input data (Cartesian coordinates and time points at transient and steady state phases).

As seen in Figure 8, there is a similar trend in the simulated air temperatures shown in Figure 2 (for the same time instant of $2000 \mathrm{~s}$ ). The inside air temperatures decrease from the top to the bottom locations, with the same problem of higher temperatures at the top right of the plane (due to insulation problems, as already mentioned). In Figure 2, it is possible to see that the domains with constant inside air temperatures show a slight tendency to lower towards the right side of the plane under consideration. In Figure 8, this tendency is much more evident for all levels of temperatures. Two conclusions can be obtained from the specifically tested refrigerator: 1) perishable products that require lower temperatures should be kept close to the right wall, up to the middle eighth of the refrigerator; 2 ) on the plane considered in the simulations, it is possible to see that the peaks of each constant temperature field are located almost on a vertical through the middle of the plane.

As can also be noted, the ANN simulates lower air temperatures at the bottom of the plane (282.7K, as compared with $286 \mathrm{~K}$ obtained with the Fluent).

As seen in Figure 8 , it is also possible to see much more detached constant temperature layers. The air temperature difference between the top and the bottom locations is larger with ANN, about 7K (as opposed to about 5K from Fluent), which means a higher simulation resolution. Also, in this figure, the spots simulated in Figure 2 have disappeared.

For the time instant of $15000 \mathrm{~s}$ in the steady state, the results of the ANN and the Fluent are not so similar. The results obtained with the ANN show much more detail about the domains of constant temperature in spite of all of them falling in the range of [279K, 280K] (in Figure 3 there are almost three different layers of constant temperatures, decreasing on average from the left to right and with a temperature difference between the top and the bottom of the same 
magnitude, about 1K). In Figure 9, a similar tendency can be seen but with many more different layers of constant temperatures. Additionally, the ANN detects the hot zone at the top right of the plane that the Fluent was not able to simulate. However, the colder zone on the top plane is not as easily identified with the ANN method, in spite of the temperatures being identical to the ones obtained with the Fluent and that measurement is approximately $279 \mathrm{~K}$.

The same kind of conclusions obtained in the transient phase can also be observed during the steady state.

Comparing with Figure 3, it is also possible to see that with the ANN, the spots of higher temperatures simulated (and not justified) by the Fluent code have disappeared with the second numerical tool.

Similar analysis can be made based on graphs obtained using different planes of the refrigerator cabin.

\section{CONCLUSIONS}

In all refrigerated spaces, it is important to understand the inside air temperature distribution to know the best places to locate products due according to their specific characteristics. One way to achieve this is to continuously monitor the inside air temperatures in several places. However, this procedure can be difficult and expensive. A more expeditious approach is to simulate the inside air temperatures using selected experimental results and adequate numerical tool packages. In this work, two different numerical tools were used to simulate the inside air temperatures in a commercial household refrigerator. One method, the commercially available Fluent code, makes use of the classic turbulent model $k-\varepsilon$ for the 3D simulations and is based on the finite volumes method, where the equations of mass conservation and momentum are solved simultaneously. The other method, the Artificial Neural Network (ANN), is based on a computational structure inspired by the biology of the neural human system including attributes such as learning, thinking, memorising, remembering, rationalising and problem solving. An ANN method 
was specially developed for the case under study. Furthermore, one of the advantages of the ANN-based approach is to use a small set of experimental measurements of air temperatures to build a tool that enables to predict the air temperature fields inside refrigeration cabins and simultaneously detecting critical variations. This avoids the necessity of exhaustive experimental tests.

Comparison of the results of the two numerical tools yields the following conclusions:

- Both are powerful numerical tools for simulation of the inside air temperatures inside refrigerated cabins.

- The absolute error (difference between the simulated and measured temperatures) obtained from the ANN $(0.8 \mathrm{~K})$ is lower than that obtained from Fluent, about $1 \mathrm{~K}$. This is not a large difference, however, with regard to the amplitude of the levels of temperatures under consideration in the refrigerator, the ANN is more precise.

- The temperature fields obtained with the ANN are more refined when compared with that of the Fluent code. During the transient phase (2000 s), the ANN was able to detect a larger difference in the simulated air temperatures when compared with the Fluent, and those differences were $7 \mathrm{~K}$ and $5 \mathrm{~K}$, respectively. In the steady phase (15000 s), there were almost no temperature differences obtained from either simulation tool.

- The temperature field patterns obtained inside the refrigerator are similar in both simulation tools.

- For the specifically tested refrigerator, it is possible to conclude that perishable products that require lower temperatures should be kept close to the right wall, up to the middle eighth of the refrigerator. On the considered plane used for the simulations, it is also possible to see that the peaks of each constant temperature field are located almost on the vertical through the middle of the plane. 
As can be observed, the air temperature differences inside the refrigerator used in the tests are not large, yet the numerical tools used in this work were able to detect them. However, in huge refrigeration storehouses, it is expected that larger variations of the air temperature will occur, which can jeopardise product conservation. Therefore, the previously mentioned numerical methods are important tools that can give a picture of the air temperature fields inside refrigeration storehouses of any dimension.

In a near future a study on the influence of products inside the refrigerator will be carried out based on further experimental measurements.

\section{AKNOWLEDGMENTS}

The authors acknowledge all of the support given by IDMEC - Polo FEUP (Institute of Mechanical Engineering - Porto Pole) to realise this work. Special acknowledgement is due to Eng. J. Matos for the experimental apparatus conducted.

\section{REFERENCES}

[1] McCulloch, W. S. and Pitts, W., "A logical Calculus of the Ideas Immanent in Nervous Activity", Bulletin of Mathematical Biophysics, Vol.5, pp.115-133, 1943. [2] Krarti, M., et al. "Estimation of Energy Saving for Building Retrofits Using Neural Networks", Journal of Solar Energy Engineering, Vol.120, pp.211-216, 1998.

[3] Aydinalp, M., et al. "Modeling of the Appliance, Lighting, and Space-cooling Energy Consumptions in the Residential Sector Using Neural Networks", Applied Energy, Vol.71, No.2, pp.87-110, 2002.

[4] Aydinalp, M., et al. "Modeling of the Space and Domestic Hot-water Heating Energy Consumption in the Residential Sector Using Neural Networks", Applied Energy, Vol.79, No.2, pp 159-178, 2004.

[5] Certiner, C. et al "Generating Hot Water by Solar Energy and Application of Neural Network", Applied Thermal Engineering, Vol.25, No.8-9, pp.1337-1348, 2005. 
[6] Ertunc, H, Hosoz, M. "Artificial Neural Network Analysis of a Refrigeration System with an Evaporative Condenser", Applied Thermal Engineering, Vol.26, pp.627-635, 2006.

[7] Arcaklioglu, E., Erisen, A., Yilmaz, R. "Artificial Neural Network Analysis of Heat Pumps Using Refrigerant Mixtures", Energy Conversion and Management, Vol.45, pp.1917-1929, 2004.

[8] Hosoz, M. and Ertunc, H. "Artificial Neural Network Analysis of an Automotive Air Conditioning System", Energy Conversion and Management, Vol.47, pp.1574-1587, 2006.

[9] Sozen, A. Rcaklioglu, E., Ozalp, M. "Artificial Neural Network Analysis of an Automotive Air Conditioning System", Applied Thermal Engineering, Vol.23, pp.937-952, 2003.

[10] Hosoz, M. and Ertunc, H. "Modelling of a Cascade Refrigeration System Using Artificial Neural Network Analysis", International Journal of Energy Research, Vol.30, pp.1200-1215, 2006.

[11] Afonso, C.F.A.; Matos, J., "Thermal Simulations and Measurements of Domestic Refrigerators-Freezers: Experimental Results", International Journal of Refrigeration, Vol.29, pp.1144-1151, 2006.

[12] Clito, A., et all, "Frigoríficos Domésticos: Valuation Térmica Frente Varios Regímenes de Funcionamento", Proceedings of the conference "Advances en Ciências y Técnicas del Frio", Vigo, Spain, 2003.

[13] Fluent User's Guide, Version 4.0, Fluent Inc., Lebanon - NH, USA, 1998.

[14] Kalogirou, S.A., "Applications of artificial neural networks in energy systems, A review", Energy Conversion \& Management, Vol.40, pp.1073-1087, 1999.

[15] Sencan, A., "Artificial intelligent methods for thermodynamic evaluation of ammonia-water refrigeration systems", Energy Conversion \& Management, Vol.47, pp.3319-3332, 2006.

[16] Sencan, A., "Performance of ammonia-water refrigeration systems using artificial neural networks", Renewable Energy, Vol.32, pp.314-328, 2007.

[17] Pacheco-Vega, A., Sen, M., Yang, K.T., McClain, R.L., "Neural network analysis of fin-tube refrigerating heat exchanger with limited experimental data", International Journal of Heat and Mass Transfer, Vol.44, pp.763-770, 2001. 
[18] Conceição António, "A Multilevel Genetic Algorithm for Optimization of Geometrically Non-Linear Stiffened Composite Structures", Structural and Multidisciplinary Optimization, Vol.24, No.5, pp.372-386, 2002.

[19] Castro, C.F., António, C.A.C. and Sousa, L.C., "Optimisation of shape and process parameters in metal forging using genetic algorithms", Journal of Materials Processing Technology, Vol.146, pp.356-364, 2004. 


\section{List of Figures}

Fig. 1 Household refrigerator used for temperature measurements.

Fig. 2 Air temperature fields $(\mathrm{K})$ in a plane parallel to the door and at a middle distance in width for the time of $2000 \mathrm{~s}$ (end of transient phase); compressor stopped. Simulation based on commercial code Fluent.

Fig.3 Air temperature fields $(K)$ in a plane parallel to the door and at a middle distance in width for the time of $15000 \mathrm{~s}$ (steady state phase); compressor about to start. Simulation based on commercial code Fluent.

Fig. 4 Topology of Artificial Neural Network (ANN).

Fig. 5 Process Element (PE) for a neuron.

Fig. 6 ANN learning process based on GA.

Fig. 7 ANN learning process, evolution of error by GA.

Fig. 8 Air temperature fields $(\mathrm{K})$ in a plane parallel to the door and at a middle distance in width for the time of $2000 \mathrm{~s}$ (end of transient phase); compressor stopped. Simulation based on developed ANN.

Fig. 9 Air temperature fields $(\mathrm{K})$ in a plane parallel to the door and at a middle distance in width for the time of $15000 \mathrm{~s}$ (steady state phase); compressor about to start. Simulation based on developed ANN. 


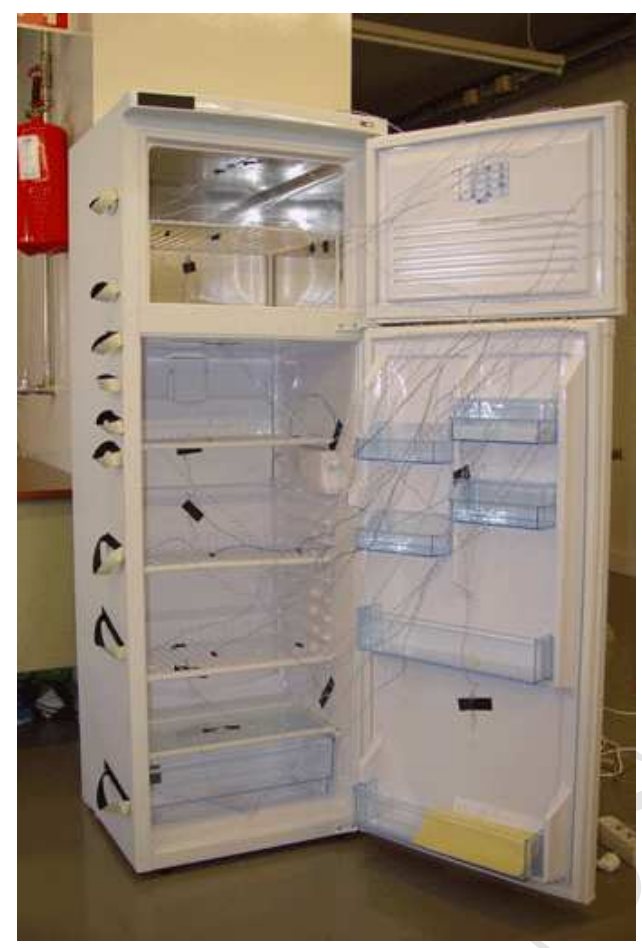

Fig. 1 Household refrigerator used for temperature measurements.
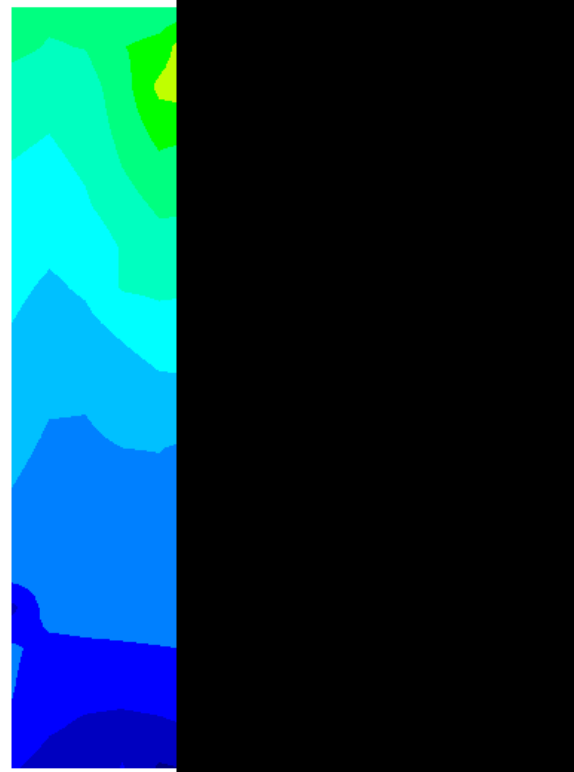

Fig. 2 Air temperature fields $(\mathrm{K})$ in a plane parallel to the door and at a middle distance in width for the time of $2000 \mathrm{~s}$ (end of transient phase); compressor stopped. Simulation based on commercial code Fluent. 

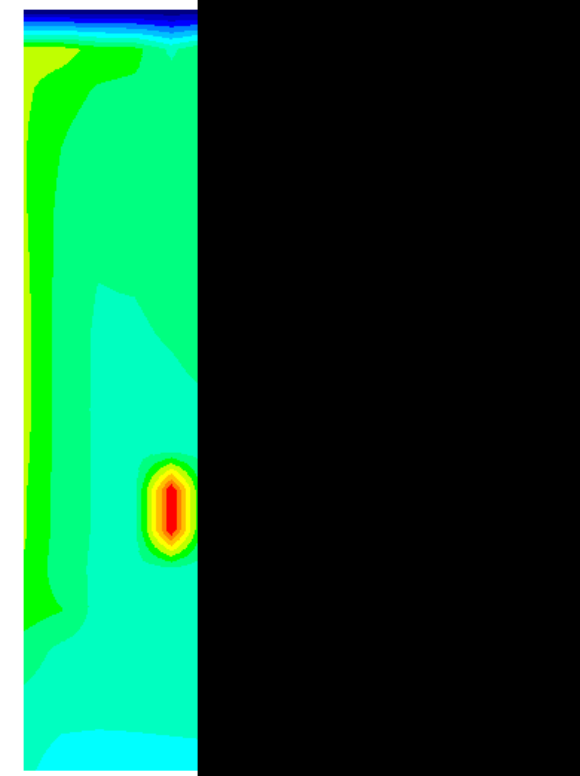

Fig.3 Air temperature fields $(\mathrm{K})$ in a plane parallel to the door and at a middle distance in width for the time of $15000 \mathrm{~s}$ (steady state phase); compressor about to start. Simulation based on commercial code Fluent.

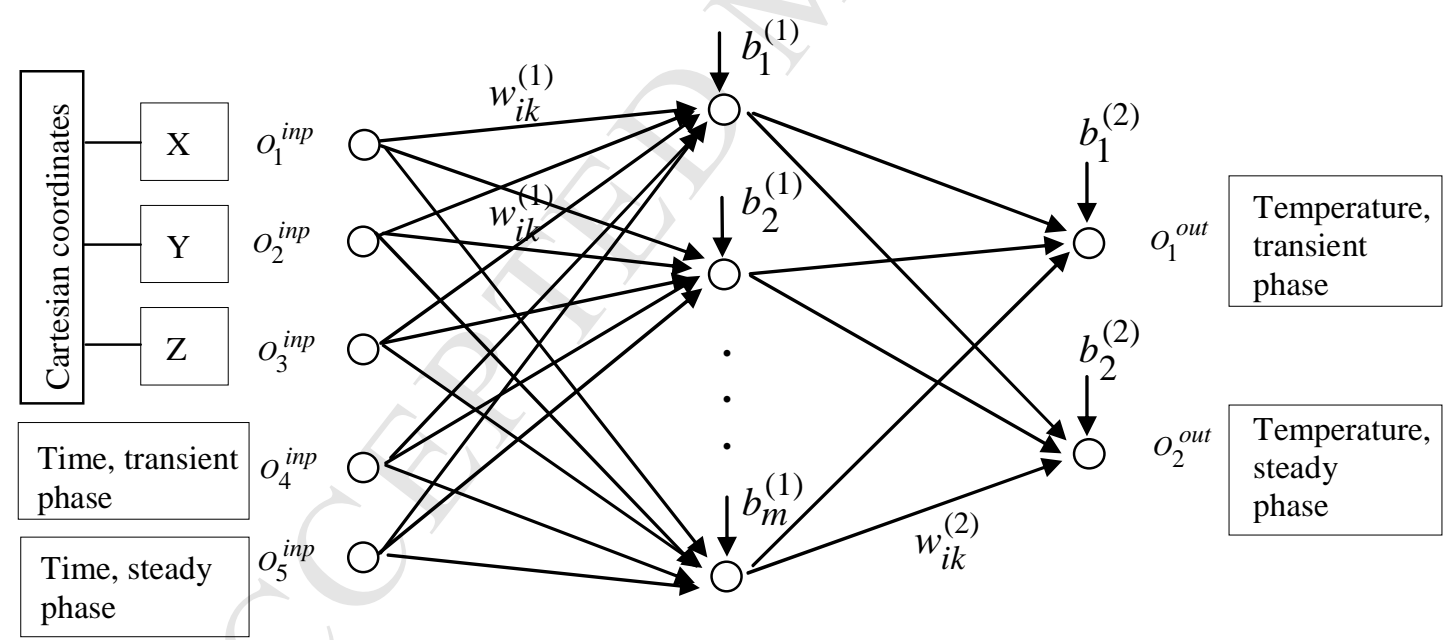

Fig. 4 Topology of Artificial Neural Network (ANN). 


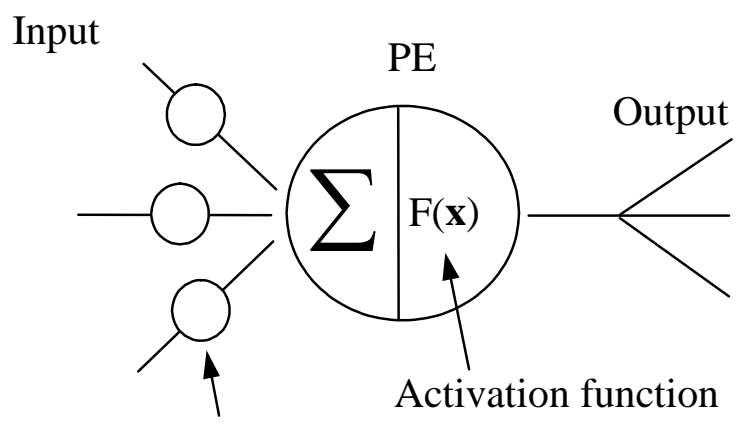

Weights

Fig. 5 Process Element (PE) for a neuron. 


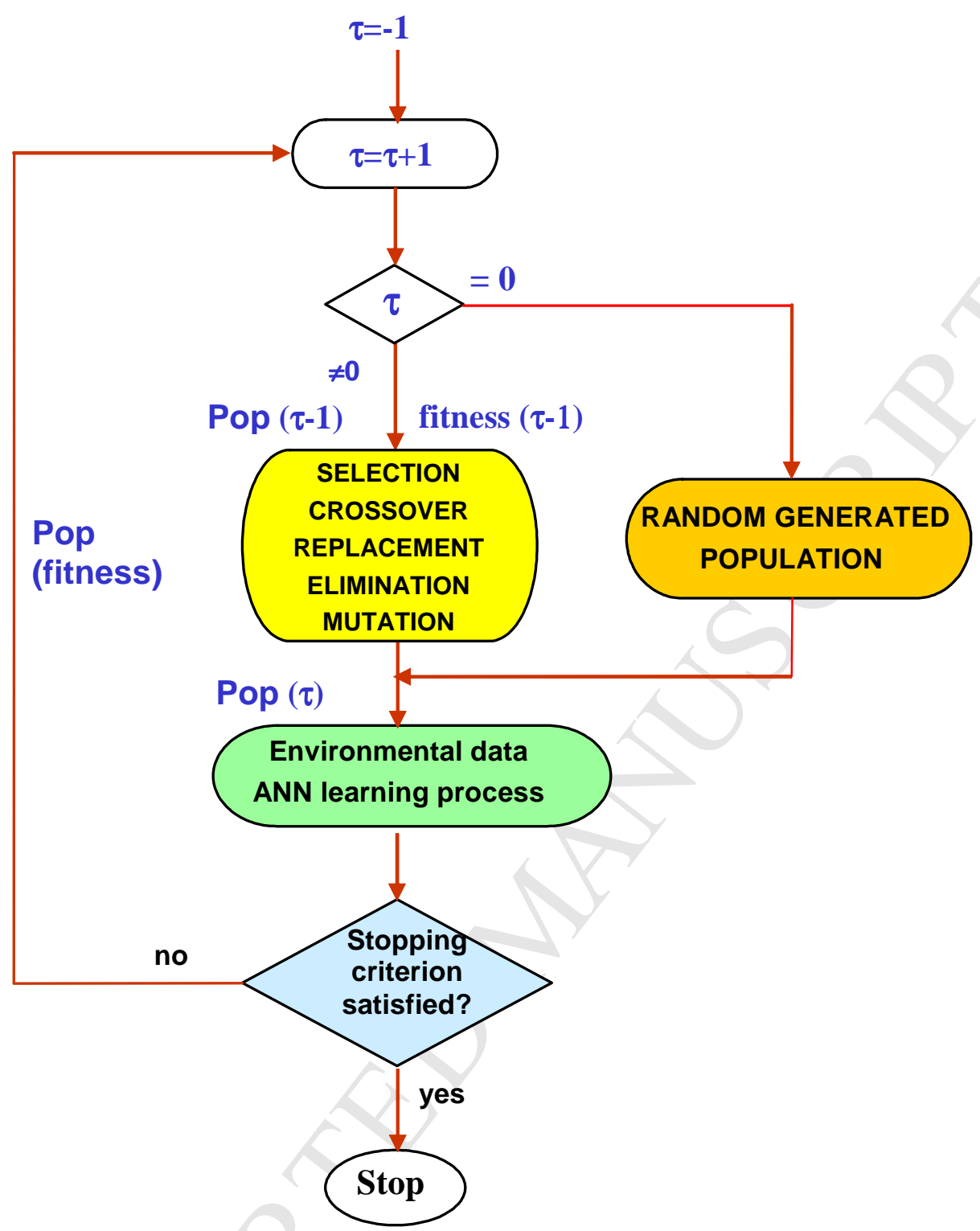

Fig. 6 ANN learning process based on GA. 


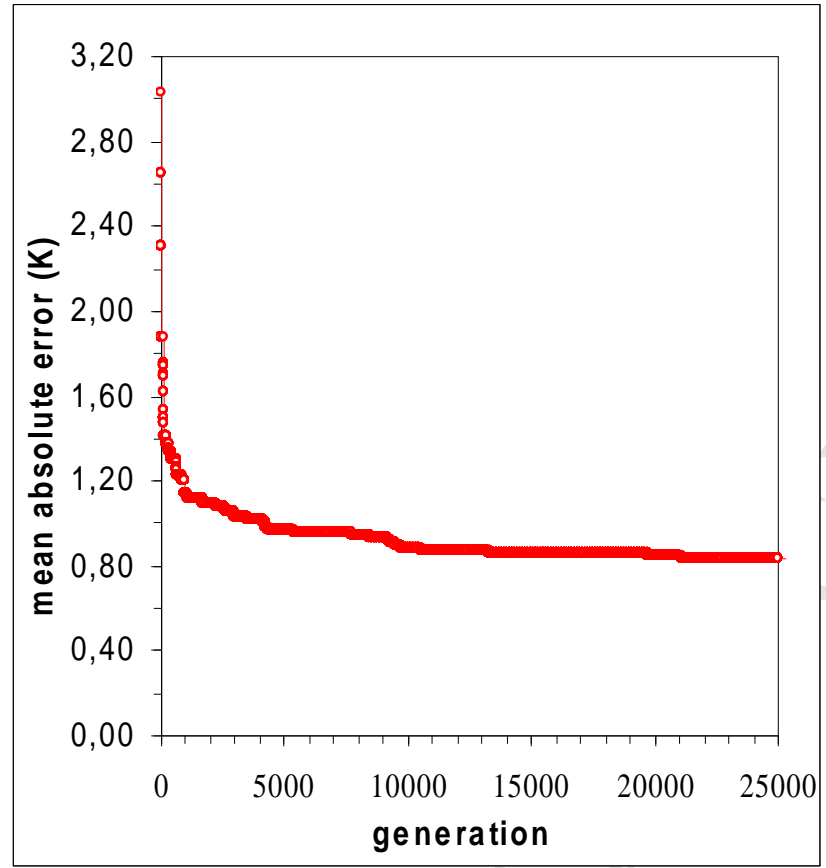

Fig. 7 ANN learning process, evolution of error by GA. 

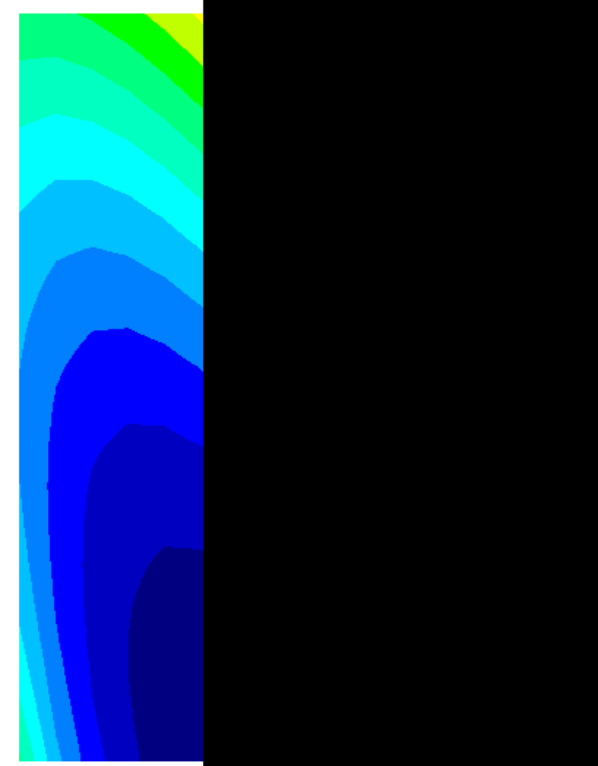

Fig. 8 Air temperature fields $(\mathrm{K})$ in a plane parallel to the door and at a middle distance in width for the time of $2000 \mathrm{~s}$ (end of transient phase); compressor stopped. Simulation based on developed ANN.
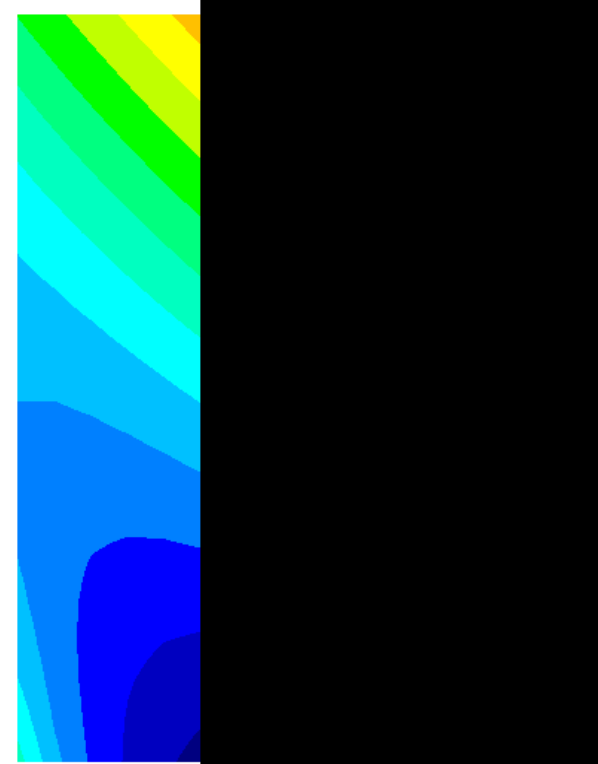

Fig. 9 Air temperature fields $(\mathrm{K})$ in a plane parallel to the door and at a middle distance in width for the time of $15000 \mathrm{~s}$ (steady state phase); compressor about to start. Simulation based on developed ANN. 
Paper submitted on Journal: Applied Thermal Engineering

Title: "Air Temperature Fields inside Refrigeration Cabins: A Comparison of Results from CFD and ANN modelling"

Authors: Carlos Conceição António and Clito F. Afonso

Reference: ATE 3351

\section{$\underline{\text { Research Highlights: }}$}

The gradients of air temperature inside domestic refrigerator are studied. Using the monitoring results an artificial neural network (ANN) is developed.

ANN results are promising when compared with the ones from other numerical tools.

ANN approach enables to predict the air temperature fields inside refrigerator.

Critical variations are detected avoiding the need for exhaustive experimental tests. 